\title{
Cross-Validation of the Arabic Mini International Neuropsychiatric Interview, Module K, for Diagnosis of Schizophrenia and the Arabic Positive and Negative Syndrome Scale
}

\author{
Rifka Chamali ${ }^{1}$ - Suhaila Ghuloum ${ }^{2}$ - David V. Sheehan ${ }^{3} \cdot$ Ziyad Mahfoud $^{4,5}$ - Arij Yehya ${ }^{1} \cdot$ Mark G. A. Opler ${ }^{6}$. \\ Anzalee Khan ${ }^{7,8} \cdot$ Samer Hammoudeh $^{1} \cdot$ Yahya Hani $^{2} \cdot$ Hassen Al-Amin $^{9}$ (D)
}

Published online: 26 August 2020

(C) The Author(s) 2019

\begin{abstract}
The Mini International Neuropsychiatric Interview, version 6, module K (MINI6-Mod-K) is a diagnostic tool to confirm schizophrenia; whereas the Positive and Negative Syndrome Scale in Schizophrenia (PANSS) is a scale to assess psychotic symptoms severity. The objective of this study was to cross-validate the diagnostic questions (categorical) from the Arabic MINI6-Mod-K with the corresponding items' scores (dimensional) from Arabic PANSS. Arab subjects $(N=101)$ were recruited from the Psychiatry Hospital in Qatar. MINI6-Mod-K was used to confirm schizophrenia diagnosis, and PANSS was administered to assess psychopathology by an independent rater. The most common symptoms according to MINI6-Mod-K were the delusions and hallucinations but the PANSS items scores $\geq 2.5$ captured more positive responses on all the diagnostic features of schizophrenia. The cut-off PANSS scores that significantly distinguished between the presence or absence of schizophrenia features on MINI6-Mod-K were mostly between 2.5 and 3.5. Multivariate linear regression showed that negative symptoms and disorganized/catatonic behaviors independently contributed to the PANSS score on positive symptoms subscale. However, none of the symptom questions in the MINI6-Mod-K were significant predictors of the PANSS negative score. Our results showed that there is very good cross-validation between the diagnostic questions of Arabic MINI6-Mod-K and the corresponding positive cut-off scores in Arabic PANSS.
\end{abstract}

Keywords Arabic PANSS · Arabic MINI6 · Schizophrenia · Psychopathology

Hassen Al-Amin

haa2019@qatar-med.cornell.edu

1 Department of Research, Weill Cornell Medicine - Qatar, Doha, Qatar

2 Department of Psychiatry, Rumailah Hospital, Hamad Medical Corporation, Doha, Qatar

3 College of Medicine, University of South Florida, Tampa, FL, USA

4 Department of Global and Public Health, Weill Cornell Medicine Qatar, Doha, Qatar

5 Department of Healthcare Policy and Research, Weill Cornell Medicine, New York, NY, USA

6 CSO-Prophase LLC, New York, NY, USA

7 NeuroCog Trials, Durham, NC, USA

8 Nathan S. Kline Institute for Psychiatric Research, Orangeburg, NY, USA

9 Department of Psychiatry, Weill Cornell Medicine-Qatar, Education City, P.O. Box 24144, Doha, Qatar

\section{Introduction}

Schizophrenia is a chronic psychotic disorder with a worldwide lifetime prevalence of $0.30 \%$ to $0.66 \%$ (McGrath et al. 2008). According to the Diagnostic and Statistical Manual for Mental Disorders (5th ed.; DSM-5) (American Psychiatric Association 2013), at least two of the following symptoms should be present to diagnose schizophrenia: delusions, hallucinations, disorganized speech, disorganized or catatonic behavior and negative symptoms. Additionally, at least one has to be from the first three symptoms. Due to the complexity of psychiatric disorders, clinical interviews are essential to confirm diagnoses in clinical and research settings. Examples of such structured interviews are the structured clinical interview for DSM (SCID) (Spitzer et al. 1992), the World Health Organization (WHO)Composite International Diagnostic Interview (CIDI) (Wittchen 1994), the Schedule for Affective Disorders and Schizophrenia (SADS) (Endicott and Spitzer 1978), and the Mini International Neuropsychiatric Interview (MINI) (Sheehan et al. 1998). 
Psychiatrists and clinicians in the US and Europe developed the MINI to establish a diagnostic interview for DSMIV psychiatric disorders (Sheehan et al. 1997; Sheehan et al. 1998). The advantages of this tool include being short, inexpensive, easy to administer, and highly sensitive and specific (Sheehan et al. 1998) to diagnostic verification. It has been translated to several languages, and its validity and reliability were examined in the Italian (Rossi et al. 2004), Japanese (Otsubo et al. 2005), Arabic (Kadri et al. 2005), Norwegian (Mordal et al. 2010) and many other countries. Specifically, the MINI has been a widely used tool in the Arab region for diagnosing postpartum depression (Hamdan and Tamim 2011), (Hamdan and Tamim 2012), alcohol abuse and dependence, (Yazbek et al. 2014) and post-traumatic stress disorder (Kazour et al. 2017). The enhanced version (MINI, version 6) for schizophrenia and psychotic disorders gives a more detailed diagnosis on the different types of psychotic disorders such as schizophrenia, schizoaffective, schizophreniform and brief psychotic disorder (Amorim et al. 1998). This corresponding module of the enhanced MINI6, Module K, (MINI6-Mod-K) was translated and linguistically validated by our team to have a gold standard diagnostic measure of psychotic disorders in our studies (Yeha et al. 2016).

Psychopathology scales have also been developed to focus on the severity of specific symptoms in people with schizophrenia, such as the Brief Psychiatric Rating Scale (BPRS) (Overall and Gorham 1988), Scale for the Assessment of Positive Symptoms (SAPS) (Andreasen 1984), Scale for the Assessment of Negative Symptoms (SANS) (Andreasen 1989), Calgary Depression Scale for Schizophrenia (CDSS) (Addington et al. 1993) and Positive and Negative Syndrome Scale (PANSS) (Kay et al. 1987). Such instruments are essential both clinically and in research, as they allow to track the changes in psychopathology symptoms in addition to assessing the efficacy of pharmacological interventions (Lindenmayer et al. 2007).

The PANSS is a widely used dimensional and typological scale that assesses the psychopathology features of schizophrenia (Kay et al. 1987). It measures the presence of positive, negative and other general symptoms in addition to their severity. It has been validated and translated into several languages such as French (Lancon et al. 1999), Chinese (Phillips et al. 1991), Spanish (Peralta Martin and Cuesta Zorita 1994) and Brazilian Portuguese (Higuchi et al. 2014) among others. The Arabic version of the PANSS was translated, culturally adapted and linguistically validated by our team (Yehya et al. 2016) to address the lack of availability of such tools in the Arabic language. It had good validity, reliability, and internal consistency and therefore proved to be a reliable measurement scale in the Arab population (Yehya et al. 2016; Yehya et al. 2017).

There are no studies that compared the diagnostic categorical scales with the dimensional measures of the severity of psychosis. Kay et al. (1991) combined the SCID interview with the PANSS to develop a two-tier "nosological-dimensional" diagnostic evaluation of psychotic symptoms. Such valid comparisons would enhance the accuracy of the psychometric measures of the combined approaches when diagnosing and monitoring patients with psychiatric disorders. The purpose of this study was to cross-validate the Arabic MINI6-Mod-K for schizophrenia and the similar symptoms of the Arabic PANSS in Arab patients with schizophrenia. The primary objective of this study was to determine the cut-off scores on the dimensional PANSS items using the answers on categorical MINI6-Mod-K as the gold standard. The second objective is to assess which of the diagnostic features in MINI6-Mod-K are contributing to the severity of the different psychotic symptoms measured by the PANSS in the Arab people with schizophrenia.

\section{Methods}

This cross-sectional study is part of a project aiming to validate the Arabic versions of psychiatric scales used in the assessment of patients with schizophrenia in the Arab population residing in Qatar (Hammoudeh et al. 2016; Hani et al. 2016; Yehya et al. 2016; Yehya et al. 2017). This paper reports a secondary analysis of the relationship between the Arabic MINI6-Mod-K and PANSS. The repeated forwardbackward translation procedure was applied to translate the MINI6-Mod-K and the PANSS from English into the Arabic language. Any inconsistencies were resolved by retaining only the translated items that perfectly matched the original MINI6-Mod-K or PANSS items after back-translating the articles into English. The details of the translation procedure were presented in the validation paper of Arabic PANSS (Yehya et al., 2016). The Institutional Review Boards of Hamad Medical Corporation and Weill Cornell Medicine in Doha, Qatar approved this study. All participants signed an informed consent form before the administration of the scales. The recruitment of subjects took place from February 2013 to November 2014.

\section{Setting and Subjects}

The study was carried out in Doha, Qatar, a country experiencing rapid economic growth and development with an increased number of expatriates from different ethnicities and nationalities residing in the country. Qataris and Arabs are the most stable populations in the state of Qatar; and they represent $15 \%$ and $13 \%$ of the total population, respectively (Qatar Statistic Authority 2010). A total of 101 Arabic schizophrenia subjects were recruited from the Department of Psychiatry, which is the only psychiatric hospital in Doha, Qatar. It has four inpatient units accommodating 70 patients with $90-95 \%$ 
occupancy rate. Additionally, ten outpatient clinics receive approximately 120 patients daily, $25 \%$ of them have the diagnosis of schizophrenia.

The inclusion criteria were (1) male or female participants, (2) between 18 and 65 years of age, (3) diagnosis of schizophrenia confirmed by the Arabic MINI6-Mod-K and according to DSM-IV-TR criteria (American Psychiatric Association 2000), (4) native Arabic speakers, and (5) able to understand and sign a written consent form. The exclusion criteria were (1) current or past alcohol or drug abuse, (2) individuals who were at a high risk to harm themselves or others, and (3) presence of any other psychiatric disorder.

The sample consisted of a total of 101 patients with schizophrenia who completed both MINI6 and PANSS by different raters. The study sample had a mean age of 35.03 (SD = 9.99) years. The majority of participants were males $(66.3 \%)$, Qataris (60.4\%), single (61.4\%), unemployed $(61.4 \%)$ and with secondary school level of education $(38.6 \%)$ (Table 1).

\section{Research Design}

We implemented all study procedures over two sessions. In the first one, a rater collected the sociodemographic, medical and psychiatric history, in addition to conducting the Arabic MINI6-Mod-K to confirm schizophrenia diagnosis. Then, within a period of maximum one week, a second independent rater, blinded for the results and information obtained by the first rater, administered the Arabic version of PANSS. The research team received formal training on how to conduct and rate both scales. For PANSS, the raters completed practice trials and obtained certification through ProPhase LLC, Rater Training Group, New York City, New York. For MINI6, the evaluators received formal training overseen by Dr. Sheehan and psychiatrists from the research team.

\section{Procedures and Measurements}

MINI6-Mod-K includes two sections, part 1 examines the presence of psychotic symptoms, rules out that these symptoms are due to a medical condition or substance abuse, assesses social and occupational dysfunctions and disability due to psychosis, and finally assesses the duration of these symptoms. Part 2 rules out the presence of a clinical mood disorder occurring concurrently with the psychotic episodes (Amorim et al. 1998). For the PANSS, the information is derived from a semi-structured Clinical Interview (SCI-PANSS) and reports of family members and treatment team (Kay 1991). Following the interview, 30 items (7 for positive symptoms, 7 for negative symptoms, and 16 for general psychopathology) are rated using a 7-point rating system (1-absent, 2-minimal, 3-mild, 4-moderate, 5-moderate-severe, 6-severe, and 7-extreme) (Kay et al. 1987). Ratings of the scale are based on information and symptoms pertaining to the previous week.
For this study, we utilized MINI6-Mod-K questions that are directly related to the main diagnostic symptoms of schizophrenia and grouped them into the following categories: delusions, hallucinations, disorganized speech, disorganized or catatonic behavior and negative symptoms. The questions are of a dichotomous type, with the possibility of either answering "yes," confirming the presence of the symptoms or "no" negating it. Specifically, we chose to compare these symptoms to their corresponding items in PANSS, by matching them according to their description in the rating criteria. Corresponding items between MINI6-Mod-K and PANSS are detailed in Table 2 .

\section{Statistical Analysis}

All analyses were performed using IBM Statistical Package for Social Sciences (SPSS) for Mac version 24 (IBM Corp, Armonk, NY, 2015). The level of significance was set at 5\%, and Bonferroni correction was used for multiple comparisons. The descriptives of the sample are summarized as means and standard deviation (SD) for the continuous measures like age, and as frequency and percentage for the categorical ones like gender. Antipsychotic dose equivalences were calculated based on chlorpromazine $100 \mathrm{mg}$ (Andreasen et al. 2010). The ordinal PANSS scores were reported through the median and interquartile range (IQR). To determine the cut-off scores on the PANSS items, we first looked at the significant differences in PANSS items scores between those who answered Yes vs. No on the dichotomous MINI6-Mod-K answers, using Wilcoxon-Mann-Whitney test. Then these comparisons were followed by Receiver Operating Characteristics (ROC) analyses, using the MINI6-Mod-K answers as the gold standard, to get the cut-off scores with the optimal sensitivity and specificity measures.

Additionally, to determine the frequency of symptomatology (measured by PANSS items groups) we transformed the ratings on PANSS items into dichotomous values and compared them to the corresponding positive diagnostic questions in MINI6-Mod-K. We started with a score $\geq 2$ as "Yes" and increased the cut-offs by one each time up to $\geq 6$ (Table 7). For each of MINI6-Mod-K questions and PANSS items, if at least one was positive, then the diagnostic category was considered present. Finally, linear regression analyses were used to assess which of the features in the MINI6-Mod-K are contributing to the various measures on PANSS total, positive, negative and general psychopathology scores. The latter scores were normally distributed. Therefore, four different linear regressions were carried out with the PANSS total, and each subscale score, as separate dependent variables; and the corresponding MINI6-Mod-K symptoms (delusions, hallucinations, disorganized speech, disorganized or catatonic behavior and negative symptoms) as independent variables (Table 2). 
Table 1 Sociodemographic characteristics of schizophrenia patients

\begin{tabular}{ll}
\hline Sociodemographic characteristics & Patients \\
\hline Mean age $(S D)$, years & $35.03(9.99)$ \\
Gender, $n(\%)$ & $67(66.3)$ \\
Male & $34(33.47)$ \\
Female & \\
Country Born, $n(\%)$ & $61(60.4)$ \\
Qatar & $40(39.6)$ \\
Non-Qatar & \\
Marital status, $n(\%)$ & $27(26.7)$ \\
Married & $62(61.4)$ \\
Single & $10(9.9)$ \\
Divorced & $1(1.0)$ \\
Widowed & \\
Education level, $n(\%)$ & $2(2.0)$ \\
Never attended school & $36(35.7)$ \\
Elementary or intermediate school & $39(38.6)$ \\
Secondary or high school & $2(2.0)$ \\
Vocational degree & $22(21.8)$ \\
College degree or postgraduate university degree & \\
Employment, $n(\%)$ & $29(28.7)$ \\
Employed & $61(61.4)$ \\
Unemployed/Other & $10(9.9)$ \\
Student & \\
\hline
\end{tabular}

$S D$ standard deviation

\section{Results}

\section{Clinical Characteristics of the Sample (Table 3)}

The mean duration of illness was about nine years, and over $28 \%$ had a history of suicidal attempts. Approximately $70 \%$ of the patients were maintained on antipsychotic treatment at the time of interview and had a mean chlorpromazine equivalent dose of $1067.1 \mathrm{mg}(\mathrm{SD}=1424.61)$ : $51 \%$ were maintained on one antipsychotic, $42 \%$ on two antipsychotics and the remaining $7 \%$ on three different types of antipsychotics. Number of admissions to the psychiatric ward ranged from 1 to 18 with a median of 3 . The mean total score on PANSS was 73.22 $(\mathrm{SD}=23.18)$ and the highest subscale score was for general psychopathology (Table 3 ).

\section{Scores on PANSS Items by MINI6-Mod-K Dichotomous Responses (Tables 4 and 5)}

Using the Wilcoxon-Mann-Whitney test, we assessed if the ordinal scores on PANSS items are different between those who answered Yes on the MINI6-Mod-K diagnostic questions vs. those who answered No. Among the MINI6-Mod-K questions (K1 to K5) related to delusions and the corresponding PANSS items, only two MINI6 diagnostic questions (K1-persecutory delusions and K2-thought broadcasting) differentiated significantly between the ratings on P1-delusions for K1 and G9unusual thought content for K2 (Table 4). For the K6-auditory

Table 2 Corresponding items of MINI6 - Module K and the PANSS

\begin{tabular}{|c|c|c|}
\hline Symptoms & Module K Items & PANSS Items \\
\hline Delusions & $\begin{array}{l}\text { K1 - Persecutory delusions } \\
\text { K2 - Thought broadcasting } \\
\text { K3 - Thought insertion } \\
\text { K4 - Ideas/delusions of reference } \\
\text { K5 - Odd beliefs/delusions }\end{array}$ & $\begin{array}{l}\text { P1 - Delusions } \\
\text { P6 - Suspiciousness and Persecution } \\
\text { G9 - Unusual thought content }\end{array}$ \\
\hline Hallucinations & $\begin{array}{l}\text { K6 - Auditory hallucinations } \\
\text { K7 - Visual hallucinations }\end{array}$ & $\begin{array}{l}\text { P3 - Hallucinatory behavior } \\
\text { G9 - Unusual thought content }\end{array}$ \\
\hline Disorganized speech & K8 - Disorganized speech & P2 - Conceptual disorganization \\
\hline Disorganized or catatonic behavior & K9 - Disorganized or catatonic behavior & $\begin{array}{l}\text { P4 - Excitement } \\
\text { G5 - Mannerisms and posturing } \\
\text { G7 - Motor Retardation }\end{array}$ \\
\hline Negative symptoms & K10 - Negative symptoms & $\begin{array}{l}\text { N1 - Blunted affect } \\
\text { N2 - Emotional withdrawal } \\
\text { N3 - Poor rapport } \\
\text { N4 - Passive/apathetic social withdrawal } \\
\text { N5 - Difficulty in abstract thinking } \\
\text { N6 - Lack of spontaneity and flow of conversation } \\
\text { N7 - Stereotyped thinking } \\
\text { G13 - Disturbance of volition }\end{array}$ \\
\hline
\end{tabular}


hallucinations, there was a significant difference in the score on P3-hallucinatory behavior; while for the visual hallucination (K7), the difference was significant only for the G9-unusual thought content (Table 5). The patients who had Yes on the K8-disorganized scores showed higher PANSS scores on the item P2-conceptual disorganization, while patients with disorganized behavior (K9) showed significantly higher scores on the item P4-Excitement. For the negative symptoms on K10, which is only one question in MINI6-Mod-K, the corresponding eight items in PANSS showed significantly higher scores for two items only, N1-blunted affect and N6-lack of spontaneity and flow of conversation (Table 5).

\section{Cut-off Scores on PANSS Items by MINI6-Mod-K Dichotomous Responses (Table 6)}

Table 6 shows the results of the ROC analyses where the areas under the curves (AUCs) were significant together with the cut-off scores (and the corresponding balanced sensitivity and specificity values) on the corresponding PANSS items using the dichotomous MINI6-Mod-K questions as the gold standard. The cut-off scores for the items on delusion where 3.5, while for the disorganized speech and behavior and negative symptoms the cut-offs were 2.5 for the corresponding PANSS items (Table 6). The items corresponding to hallucinations on MINI6-Mod-K ranged from 1.5-3.5 where P3-hallucinatory behavior was the highest and G9-unusual thought content was the lowest when contrasted with the presence of auditory hallucinations. The balanced sensitivity and specificity values for

Table 3 Clinical features of patients with schizophrenia

\begin{tabular}{ll}
\hline Variables & Mean (SD) \\
\hline Age of symptoms onset & $22.97(8.42)$ \\
Number of hospitalizations & $3.93(3.07)$ \\
Duration of illness, years & $9.14(8.76)$ \\
Antipsychotics dose equivalence, mg & $1067.11(1424.61)$ \\
& $\mathrm{N}(\%)$ \\
Suicide Attempt & \\
$\quad$ No & $64(63.4 \%)$ \\
$\quad$ Yes & $29(28.7 \%)$ \\
Aggressive behavior & \\
No & $58(57.4 \%)$ \\
Yes & $37(36.6 \%)$ \\
& Mean (SD) \\
PANSS Total & $73.22(23.18)$ \\
PANSS Positive & $19.88(7.07)$ \\
PANSS Negative & $18.76(8.71)$ \\
\hline
\end{tabular}

Antipsychotics dose equivalence was based on chlorpromazine $100 \mathrm{mg}$. $S D$, standard deviation all the corresponding cut-off scores were good ranging from 0.435 to 0.739 (Table 6).

\section{The Frequency of Module K Items and PANSS Symptom Groups (Table 7)}

We assessed the frequency of the diagnostic features in MINI6-Mod-K and the corresponding PANSS items by grouping the components into symptom groups as described in Table 2. Table 7 shows the frequency of the presence or absence of each of the MINI6-Mod-K psychotic symptoms together with any of the corresponding items on PANSS as per Table 1. MINI6-Mod-K showed that delusions and hallucinations were the most common symptoms in this sample. The ratings on PANSS symptoms groups captured the same frequencies as MINI6-Mod-K Delusions at score $\geq 2$ and for Hallucinations at scores $\geq 3$. For the remaining symptoms (disorganized speech, disorganized and catatonic behavior and negative symptoms) they were not commonly present as per MINI6-Mod-K responses. However, the scores on these PANSS symptoms groups were more frequently positive at scores between 2 and 3 (Table 7).

\section{Multiple Linear Regressions (Table 8)}

Four multiple linear regression analyses were performed to predict the PANSS total and subscales scores using the MINI6-Mod-K symptoms as independent predictors: delusions, hallucinations, disorganized speech, disorganized or catatonic behavior and negative symptoms (Table 8). For the PANSS positive subscale, the model was a good fit $\left(R^{2}=0.20\right)$. The MINI6-Mod-K responses on disorganized/catatonic behavior and negative symptoms were significant predictors of the PANSS positive subscale score. The model using the PANSS negative subscale as a dependent variable was not a good fit $\left(R^{2}=\right.$ 0.04), and none of the MINI6-Mod-K diagnostic questions were significant predictors. For the subscale score on PANSS general psychopathology the model was good $\left(R^{2}=0.13, p<0.05\right)$ and the responses on the MINI6Mod-K questions on hallucinations and disorganized/ catatonic were significant predictors. Regarding the PANSS total score, the model was a good fit $\left(R^{2}=0.12\right.$, $p<0.05)$ and again the disorganized / catatonic behavior was a significant predictor of the PANSS total score.

\section{Discussion}

This study aimed to determine the cut-off scores on the dimensional PANSS items that differentiate the categorical MINI-Mod-K diagnostic features of schizophrenia and to assess the relationships between the diagnostic 
Table 4 Scores on PANSS items by MINI6-Mod-K (K1-K5) dichotomous responses

\begin{tabular}{|c|c|c|c|c|c|}
\hline \multirow{2}{*}{$\begin{array}{l}\text { MINI6-Mod-K questions } \\
\text { PANSS items scores }\end{array}$} & \multicolumn{2}{|l|}{ No } & \multicolumn{2}{|l|}{ Yes } & \multirow[b]{2}{*}{ P value } \\
\hline & Median & IQR & Median & IQR & \\
\hline \multicolumn{6}{|l|}{ K1 - Persecutory Delusions } \\
\hline P1 - Delusions & 3.00 & 2.00 & 4.00 & 2.00 & $0.015^{*}$ \\
\hline P6 - Suspiciousness & 3.00 & 1.00 & 3.00 & 3.00 & 0.117 \\
\hline G9 - Unusual thought content & 2.00 & 2.00 & 2.00 & 2.00 & 0.868 \\
\hline \multicolumn{6}{|l|}{ K2 - Thought broadcasting } \\
\hline P1 - Delusions & 3.00 & 2.00 & 4.00 & 2.00 & 0.183 \\
\hline P6 - Suspiciousness & 3.00 & 2.00 & 3.00 & 2.00 & 0.916 \\
\hline G9 - Unusual thought content & 2.00 & 2.00 & 3.00 & 2.00 & $0.021 *$ \\
\hline \multicolumn{6}{|l|}{ K3 - Thought insertion } \\
\hline P1 - Delusions & 4.00 & 3.00 & 4.00 & 2.00 & 0.131 \\
\hline P6 - Suspiciousness & 3.00 & 2.00 & 3.00 & 3.00 & 0.369 \\
\hline G9 - Unusual thought content & 2.00 & 2.00 & 2.00 & 3.00 & 0.071 \\
\hline \multicolumn{6}{|l|}{ K4 - Ideas/delusions of reference } \\
\hline P1 - Delusions & 4.00 & 3.00 & 4.00 & 3.00 & 0.072 \\
\hline P6 - Suspiciousness & 3.00 & 2.00 & 3.00 & 3.00 & 0.660 \\
\hline G9 - Unusual thought content & 2.00 & 2.00 & 3.00 & 3.00 & 0.102 \\
\hline \multicolumn{6}{|l|}{ K5 - Odd beliefs/delusions } \\
\hline P1 - Delusions & 4.00 & 2.00 & 3.00 & 3.00 & 0.112 \\
\hline P6 - Suspiciousness & 3.00 & 3.00 & 3.00 & 2.00 & 0.164 \\
\hline G9 - Unusual thought content & 2.50 & 2.00 & 2.00 & 2.00 & 0.253 \\
\hline
\end{tabular}

$I Q R$ interquartile range

categorical questions of MINI6-Mod-K for schizophrenia with the corresponding dimensional measures from PANSS items. In the following sections we will summarize each aspect of our findings followed by a discussion of its significance:

\section{MINI6-Mod K Responses and Ratings on PANSS Items}

Our results showed that when comparing each diagnostic question in MINI6-Mod-K with its corresponding item in PANSS, not all the PANSS items can differentiate well the various categorical answers in MINI6-Mod-K (Tables 4 and $5)$. The patients who had positive responses on K3-K5 (Table 4) received similar ratings on the corresponding PANSS items but some of the common items like P1Delusion were significantly higher in those who were positive on K1-Persecutory Delusion; and G9-Unusual thought content differentiated well those with positive K2-Thought broadcasting. It seems that there are no specific Arabic PANSS items that capture well the specifics of MINI items K3-K5. On the other hand, there are no specific questions in MINI6Mod-K that assess all the different features of negative symptoms in the PANSS items (N1-N7 and G13) and thus only two items (N1 and N6) showed significantly higher ratings in those who were positive on the only MINI6 K10 question (Table 5).

\section{Cut-off Scores of PANSS Items}

The majority of the cut-off scores that showed significant differentiation (P1, P2, P3, P4, G9, N1, and N6) ranged from 2.5-3.5 with adequate sensitivity and specificity (Table 6). MINI6-Mod$\mathrm{K}$ and PANSS have different purposes and have been designed accordingly. The former was validated against the SCID and CIDI with the aim of deriving a categorical dimension (Sheehan et al. 1998; Sheehan et al. 1997), whereas the latter was based on dimensional scaling using the BPRS to derive functional dimensions of schizophrenia (Kay et al. 1987). However, since both MINI6-Mod-K and the PANSS scales are centered on schizophrenia, they are therefore assessing the same core symptoms. Nevertheless, there are no studies that crossvalidated these two levels of measures (categorical and dimensional) in the scales used (English or other languages) to diagnose schizophrenia and assess the severity of psychosis. In fact, Kay et al. (1991) introduced this approach by developing the SCIDPANSS (SCID is the structured clinical interview for DSM and not the semi-structured clinical interview for PANSS, SCIPANSS), with the aim of combining general diagnostic criteria with dimensional ratings (from SCID) to get a categorical psychotic diagnosis with specific rating criteria (from PANSS) to assess severity of symptoms (Kay et al. 1991). Furthermore, in this SCID-PANSS a score of 1-absent and 2-subthreshold, corresponded to a score of 1-absent and 2-minimal in the 
Table 5 Scores on PANSS items by MINI6-Mod-K (K6-K10) dichotomous responses

\begin{tabular}{|c|c|c|c|c|c|}
\hline \multirow{2}{*}{$\begin{array}{l}\text { MINI6-Mod-K questions } \\
\text { PANSS items scores }\end{array}$} & \multicolumn{2}{|l|}{ No } & \multicolumn{2}{|l|}{ Yes } & \multirow[b]{2}{*}{$P$ value } \\
\hline & Median & IQR & Median & IQR & \\
\hline \multicolumn{6}{|l|}{ K6 - Auditory Hallucinations } \\
\hline P3 - Hallucinatory behavior & 3.00 & 2.00 & 4.00 & 2.00 & $0.005^{*}$ \\
\hline G9 - Unusual thought content & 2.00 & 2.00 & 2.00 & 3.00 & $0.026^{*}$ \\
\hline \multicolumn{6}{|l|}{ K7 - Visual Hallucinations } \\
\hline P3 - Hallucinatory behavior & 4.00 & 3.00 & 4.00 & 2.00 & 0.630 \\
\hline G9 - Unusual thought content & 2.00 & 2.00 & 3.00 & 2.00 & $0.007 *$ \\
\hline \multicolumn{6}{|l|}{ K8 - Disorganized speech } \\
\hline P2 - Conceptual disorganization & 2.00 & 3.00 & 3.00 & 2.00 & $0.009^{*}$ \\
\hline \multicolumn{6}{|l|}{ K9 - Disorganized / catatonic behavior } \\
\hline P4 - Excitement & 2.00 & 2.00 & 3.00 & 3.00 & $0.015^{*}$ \\
\hline G5 - Mannerisms and posturing & 1.00 & 1.00 & 2.00 & 2.00 & 0.054 \\
\hline G7 - Motor Retardation & 1.00 & 1.00 & 1.00 & 1.00 & 0.409 \\
\hline \multicolumn{6}{|l|}{ K10 - Negative Symptoms } \\
\hline N1 - Blunted affect & 2.00 & 2.00 & 3.00 & 2.00 & $0.043^{*}$ \\
\hline N2 - Emotional withdrawal & 2.00 & 3.00 & 3.00 & 2.00 & 0.196 \\
\hline N3 - Poor rapport & 2.00 & 2.00 & 2.00 & 2.00 & 0.061 \\
\hline N4 - Passive/apathetic social withdrawal & 2.00 & 2.00 & 3.00 & 2.00 & 0.128 \\
\hline N5 - Difficulty in abstract thinking & 3.00 & 4.00 & 3.00 & 3.00 & 0.313 \\
\hline N6 - Lack of spontaneity and flow of conversation & 2.00 & 3.00 & 3.00 & 2.00 & $0.030 *$ \\
\hline N7 - Stereotyped thinking & 2.00 & 2.00 & 3.00 & 2.00 & 0.587 \\
\hline G13 - Disturbance of volition & 2.00 & 2.00 & 2.00 & 2.00 & 0.818 \\
\hline
\end{tabular}

$I Q R$ interquartile range
PANSS. Accordingly, a score of 3-threshold in the SCID-PANSS compared to any score from 3-mild to 7-extreme in the PANSS
(Kay et al. 1991). Our results confirmed this distinction also where all the diagnostic features of schizophrenia in Arabic
Table 6 The cut-off scores of the PANSS items with significant AUC

\begin{tabular}{|c|c|c|c|c|c|}
\hline & AUC & P value & $\begin{array}{l}\text { Cut- } \\
\text { off }\end{array}$ & Sensitivity & Specificity \\
\hline \multicolumn{6}{|l|}{ K1 - Persecutory Delusions } \\
\hline P1 - Delusions & 0.658 & $0.017 *$ & 3.5 & 0.640 & 0.654 \\
\hline \multicolumn{6}{|l|}{ K2 - Thought broadcasting } \\
\hline G9 - Unusual thought content & 0.632 & $0.025^{*}$ & 2.5 & 0.510 & 0.653 \\
\hline \multicolumn{6}{|l|}{ K6 - Auditory Hallucinations } \\
\hline P3 - Hallucinatory behavior & 0.688 & $0.006 *$ & 3.5 & 0.592 & 0.739 \\
\hline G9 - Unusual thought content & 0.649 & $0.031 *$ & 1.5 & 0.743 & 0.435 \\
\hline \multicolumn{6}{|l|}{ K7 - Visual Hallucinations } \\
\hline G9 - Unusual thought content & 0.654 & $0.009 *$ & 2.5 & 0.535 & 0.661 \\
\hline \multicolumn{6}{|l|}{ K8 - Disorganized speech } \\
\hline P2 - Conceptual disorganization & 0.666 & $0.010^{*}$ & 2.5 & 0.759 & 0.515 \\
\hline \multicolumn{6}{|c|}{ K9 - Disorganized / catatonic behavior } \\
\hline P4 - Excitement & 0.687 & $0.022 *$ & 2.5 & 0.600 & 0.737 \\
\hline \multicolumn{6}{|l|}{ K10 - Negative Symptoms } \\
\hline N1 - Blunted affect & 0.626 & $0.050 *$ & 2.5 & 0.517 & 0.676 \\
\hline N6 - Lack of spontaneity and & 0.635 & $0.036^{*}$ & 2.5 & 0.724 & 0.632 \\
\hline
\end{tabular}

$A U C$, area under the curve 
Table 7 Frequency of the dichotomous responses on the MINI - Module K questions and PANSS items dichotomous scores that correspond to the five main symptoms of schizophrenia

\begin{tabular}{|c|c|c|c|c|c|c|c|}
\hline & & $\begin{array}{l}\text { Module K } \\
\text { N (\%) }\end{array}$ & $\begin{array}{l}\text { PANSS } \geq 2 \\
N(\%)\end{array}$ & $\begin{array}{l}\mathrm{PANSS} \geq 3 \\
\mathrm{~N}(\%)\end{array}$ & $\begin{array}{l}\mathrm{PANSS} \geq 4 \\
\mathrm{~N}(\%)\end{array}$ & $\begin{array}{l}\text { PANSS } \geq 5 \\
N(\%)\end{array}$ & $\begin{array}{l}\mathrm{PANSS} \geq 6 \\
\mathrm{~N}(\%)\end{array}$ \\
\hline \multirow[t]{2}{*}{ Delusions } & No & $5(5 \%)$ & $4(4 \%)$ & $14(13.9 \%)$ & $34(33.7 \%)$ & $59(58.4 \%)$ & $78(77.2 \%)$ \\
\hline & Yes & $96(95 \%)$ & $97(96 \%)$ & $87(86.1 \%)$ & $66(65.3 \%)$ & $42(41.6 \%)$ & $23(22.8 \%)$ \\
\hline \multirow[t]{2}{*}{ Hallucinations } & No & $17(16.8 \%)$ & $9(8.9 \%)$ & $20(19.8 \%)$ & $42(41.6 \%)$ & $64(63.4 \%)$ & $92(91.1 \%)$ \\
\hline & Yes & $84(83.2 \%)$ & $92(91.1 \%)$ & $81(80.2 \%)$ & $59(58.4 \%)$ & $37(36.6 \%)$ & $9(8.9 \%)$ \\
\hline \multirow[t]{2}{*}{ Disorganized speech } & No & $68(67.3 \%)$ & $20(19.8 \%)$ & $43(42.6 \%)$ & $65(64.4 \%)$ & $84(83.2 \%)$ & $92(91.1 \%)$ \\
\hline & Yes & $29(28.7 \%)$ & $81(80.2 \%)$ & $58(57.4 \%)$ & $36(35.6 \%)$ & $17(16.8 \%)$ & $9(8.9 \%)$ \\
\hline \multirow{2}{*}{$\begin{array}{l}\text { Disorganized/ } \\
\text { catatonic behavior }\end{array}$} & No & $81(80.2 \%)$ & $16(15.8 \%)$ & $46(45.5 \%)$ & $78(77.2 \%)$ & $91(90.1 \%)$ & $97(96 \%)$ \\
\hline & Yes & $15(14.9 \%)$ & $85(84.2 \%)$ & $55(54.5 \%)$ & $23(22.8 \%)$ & $10(9.9 \%)$ & $4(4 \%)$ \\
\hline \multirow[t]{2}{*}{ Negative symptoms } & No & $68(67.3 \%)$ & $8(7.9 \%)$ & $20(19.8 \%)$ & $39(38.6 \%)$ & $59(58.4 \%)$ & $77(76.2 \%)$ \\
\hline & Yes & $29(28.7 \%)$ & $93(92.1 \%)$ & $81(80.2 \%)$ & $62(61.4 \%)$ & $42(41.6 \%)$ & $24(23.8 \%)$ \\
\hline
\end{tabular}

MINI6-Mod-K were captured at the cutoff scores greater than 2.5 on the Arabic PANSS items (Table 6).

Few studies assessed the relevance of PANSS rating scores and its significance clinically. In some clinical trials, a minimum score in PANSS items is an eligibility criterion, where a minimum score of 4-moderate is required. This cut-off score is chosen as an indication that the patient is experiencing florid psychotic symptoms (Leucht et al. 2005). Furthermore, when assessing remission in schizophrenia, patients were considered in remission according to PANSS items if the score is less or equal to 3-mild (Andreasen et al. 2005; Madhivanan et al. 2017; van Os et al. 2006). Therefore, proposing that a score of 4 and above suggests that the symptom is still active and clinically significant. Thus, our cross-validation results on the Arabic PANSS and MINI6-Mod-K agree with the other international studies on schizophrenia and can be used in clinical setting and research trials for precise diagnosis and valid assessment of severity of symptoms. The patients in our study were relatively stable and have been maintained on antipsychotics for several years. However, a study by Anderson et al. (2017) analyzing data on PANSS from 11 clinical trials (applying the post-treatment bifactor item response theory model) reported that there was disparity between the remission rates as per DSM criteria and the symptom relief measures when the PANSS score were $<2$. About two thirds of the patients achieving remission had several items from PANSS with no relief of symptoms as the scores were above 3 . Thus, the authors concluded that the remission criteria should not be equated with PANSS symptoms relief measures and that the two methods might be identifying two separate subsets of patients with different measures on the various PANSS items (Anderson et al. 2017).

\section{Frequency of the MINI6-Mod-K Diagnostic Features and Corresponding PANSS Clusters}

It is worth noting that the frequency of positive findings on the Delusions (95\%) and Hallucinations (83.2\%) module K questions were high (Table 7) while those for Disorganized speech/ behavior, Catatonia, and Negative symptoms (14.9-28.7\%) were low. When clustering PANSS items into the five main schizophrenia symptom categories, the Arabic PANSS

Table 8 Multiple linear regression models of the relation between Module K symptoms and PANSS scores

\begin{tabular}{|c|c|c|c|c|c|c|c|c|}
\hline & \multicolumn{2}{|c|}{ PANSS Positive } & \multicolumn{2}{|c|}{ PANSS Negative } & \multicolumn{2}{|c|}{ PANSS General Psychopathology } & \multicolumn{2}{|c|}{ PANSS Total } \\
\hline & $B$ & $95 \% \mathrm{CI}$ & $B$ & $95 \% \mathrm{CI}$ & $B$ & $95 \% \mathrm{CI}$ & $B$ & $95 \% \mathrm{CI}$ \\
\hline Delusions & 3.03 & -3.05 to 9.11 & -1.20 & -9.45 to 7.03 & 8.14 & -2.31 to 18.59 & 9.94 & -11.16 to 31.09 \\
\hline Hallucinations & 2.87 & -0.86 to 6.60 & 2.50 & -2.56 to 7.55 & 6.55 & 0.15 to $12.96 *$ & 11.92 & -1.03 to 24.87 \\
\hline Disorganized speech & 1.29 & -2.15 to 4.73 & 2.10 & -2.56 to 6.77 & -0.65 & -6.56 to 5.26 & 2.74 & -9.21 to 14.70 \\
\hline Disorganized / catatonic behavior & 8.64 & 4.44 to $12.85 * *$ & 0.64 & -5.06 to 6.35 & 10.78 & 3.55 to $18.01 *$ & 20.07 & 5.45 to $34.68^{*}$ \\
\hline Negative Symptoms & -3.67 & -6.73 to $-0.21 *$ & 1.49 & -2.93 to 5.92 & -3.01 & -8.62 to 2.60 & -4.99 & -16.32 to 6.34 \\
\hline R-squared & 0.20 & & 0.04 & & 0.13 & & 0.12 & \\
\hline$F$ change & $4.55^{* *}$ & & 0.82 & & $2.61 *$ & & $2.32 *$ & \\
\hline
\end{tabular}

${ }^{*} p<0.05 ; * * p<0.01 ; B$, unstandardized coefficient 
showed similar frequencies with the Arabic-MINI at a PANSS score $\geq 2$ and $\geq 3$ for delusions and hallucinations respectively thus suggesting that at these cut-off scores the PANSS items capture the precise symptom categories as the MINI6-Mod-K. However, for the less frequent items as measured by MINI6Mod-K (disorganized speech, disorganized and catatonic behavior and negative symptoms), the PANSS clusters (starting from scores 2 and 3) for these diagnostic features captured more related positive features than the dichotomous MINI6Mod-K items. Furthermore, it should be noted that disorganized behavior and negative symptoms are assessed differently in MINI6-Mod-K and PANSS. Only one question is contributing to each of these symptoms in MINI6-Mod-K (K9 and $\mathrm{K} 10)$, whereas there are three PANSS items (P4, G5, and G7) for the K9 and eight items (N1-N7 and G13) to evaluate the presence of negative symptoms in PANSS. This higher number of PANSS items to assess a diagnostic feature would give a better comprehensive assessment than one question or one observation.

\section{Predictors of PANSS Total and Subscales Scores}

Furthermore, when examining if any schizophrenia MINI6Mod-K diagnostic features predict PANSS severity, we found that disorganized or catatonic behavior in schizophrenia predicted more severe symptomatology with an increase in PANSS positive, general psychopathology, and total scores (Table 8). This suggests that the presence of disorganized or catatonic symptoms is a marker of higher symptom severity. A study assessing the relationship between symptom dimensions and functioning also found that disorganized symptoms had the highest effect on functioning and prevented patients from achieving remission (Ortiz et al. 2015). Furthermore, in a study assessing treatment response of disorganized schizophrenia inpatients, it was found that these patients were more treatment-resistant in comparison with paranoid schizophrenia inpatients and had more severe PANSS scores (Ortiz et al. 2013). Thus, the Arabic MINI-Mod-K and PANSS can capture this important feature in the Arab schizophrenia patients and thus be a reliable measure to diagnose and properly assess this group of patients. We should add that the R-squared are small and thus their contributions to the variance in the PANSS scores is minimal but significant. Thus, there are probably other features, not captured by MINI6-Mod-K questions that are contributing to the psychopathology as measured by PANSS scores.

\section{Limitations}

Our study has several strengths like the validation of the Arabic MINI6-Mod-K and PANSS in the same sample, the research design and the proper training of the raters. However, few limitations can affect the significance of our results. First, the sample size is not large enough to capture a better representation of the different Arabic cultures and more heterogeneity of the different schizophrenia symptoms. Second, there are more males in this sample, and thus there could be a gender effect that biases the results. Third, tools were translated to formal Arabic which is the formal language of all Arab countries but is not the usual dialect used by different Arabic cultures, and thus the level of education (and knowledge of formal Arabic) might affect the communication styles of the Arab patients.

\section{Conclusions}

In conclusion, our results demonstrated significant relationships between the Arabic MINI6-Mod-K diagnostic features of schizophrenia and the clinically relevant cutoff scores from the dimensional ratings on the corresponding Arabic PANSS items. It also showed that the different diagnostic features of schizophrenia are differentially contributing to the variations in the different psychopathology subscales in the Arab subjects with schizophrenia. This cross-validation study gives reassurance that the valid Arabic MINI-Mod-K and Arabic PANSS are providing complementary assessments to cover the categorical and dimensional aspects of schizophrenia and that their results can be compared safely with other international studies using these instruments.

Acknowledgements Open Access funding provided by the Qatar National Library.

Funding This study was part of a project funded solely by Qatar National Research Fund (QNRF) granted to Dr. Hassen Al-Amin (NPRP 4-268-3086). QNRF did not have any additional role in the study design, data collection and analysis, interpretation of data, decision to publish, or preparation of the manuscript.

\section{Compliance with Ethical Standards}

Ethical Approval All procedures performed in studies involving human participants were in accordance with the ethical standards of the institutional and/or national research committee and with the 1964 Helsinki declaration and its later amendments or comparable ethical standards.

Informed Consent Informed consent was obtained from all individual participants included in the study.

Competing Interests All the authors have no competing interests.

Open Access This article is distributed under the terms of the Creative Commons Attribution 4.0 International License (http:// creativecommons.org/licenses/by/4.0/), which permits unrestricted use, distribution, and reproduction in any medium, provided you give appropriate credit to the original author(s) and the source, provide a link to the Creative Commons license, and indicate if changes were made. 


\section{References}

Addington, D., Addington, J., \& Maticka-Tyndale, E. (1993). Assessing depression in schizophrenia: The Calgary depression scale. The British Journal of Psychiatry. Supplement, (22), 39-44.

American Psychiatric Association. (2000). Diagnostic and statistical manual of mental disorders (4th ed.). DC: Washington.

American Psychiatric Association. (2013). Diagnostic and statistical manual of mental disorders (5th ed.). VA: Arlington.

Amorim, P., Lecrubier, Y., Weiller, E., Hergueta, T., \& Sheehan, D. (1998). DSM-IH-R psychotic disorders: Procedural validity of the MINI international neuropsychiatric interview (MINI). Concordance and causes for discordance with the CIDI. European Psychiatry, 13(1), 26-34. https://doi.org/10.1016/S0924-9338(97) 86748-X.

Anderson, A. E., Reise, S. P., Marder, S. R., Mansolf, M., Han, C., \& Bilder, R. M. (2017). Disparity between general symptom relief and remission criteria in the positive and negative syndrome scale (PANSS): A post-treatment bifactor item response theory model. Innov Clin Neurosci, 14(11-12), 41-53.

Andreasen, N. C. (1984). The scale for the assessment of positive symptoms (SAPS). University of Iowa.

Andreasen, N. C. (1989). The scale for the assessment of negative symptoms (SANS): Conceptual and theoretical foundations. The British Journal of Psychiatry. Supplement, (7), 49-58.

Andreasen, N. C., Carpenter, W. T., Jr., Kane, J. M., Lasser, R. A., Marder, S. R., \& Weinberger, D. R. (2005). Remission in schizophrenia: Proposed criteria and rationale for consensus. The American Journal of Psychiatry, 162(3), 441-449. https://doi.org/ 10.1176/appi.ajp.162.3.441.

Andreasen, N. C., Pressler, M., Nopoulos, P., Miller, D., \& Ho, B. C. (2010). Antipsychotic dose equivalents and dose-years: A standardized method for comparing exposure to different drugs. Biological Psychiatry, 67(3), 255-262. https://doi.org/10.1016/j.biopsych. 2009.08.040.

Endicott, J., \& Spitzer, R. L. (1978). A diagnostic interview: The schedule for affective disorders and schizophrenia. Archives of General Psychiatry, 35(7), 837-844.

Hamdan, A., \& Tamim, H. (2011). Psychosocial risk and protective factors for postpartum depression in the United Arab Emirates. Archives of Women's Mental Health, 14(2), 125-133. https://doi. org/10.1007/s00737-010-0189-8.

Hamdan, A., \& Tamim, H. (2012). The relationship between postpartum depression and breastfeeding. International Journal of Psychiatry in Medicine, 43(3), 243-259.

Hammoudeh, S., Ghuloum, S., Mahfoud, Z., Opler, M., Khan, A., Yehya, A., et al. (2016). Reliability, validity and factorial structure of the Arabic version of the international suicide prevention trial (InterSePT) scale for suicidal thinking in schizophrenia patients in Doha, Qatar. BMC Psychiatry, 16(1), 437, doi:10.1186/s12888-016$1155-4$.

Hani, Y., Ghuloum, S., Mahfoud, Z., Opler, M., Khan, A., Yehya, A., et al. (2016). Validation of the Arabic Version of Calgary Depression Scale for Schizophrenia. PLoS One, 11(9), e 0162304, doi:10.1371/ journal.pone.0162304.

Higuchi, C. H., Ortiz, B., Berberian, A. A., Noto, C., Cordeiro, Q., Belangero, S. I., Pitta, J. C., Gadelha, A., \& Bressan, R. A. (2014). Factor structure of the positive and negative syndrome scale (PANSS) in Brazil: Convergent validation of the Brazilian version. Revista Brasileira de Psiquiatria, 36(4), 336-339. https://doi.org/ 10.1590/1516-4446-2013-1330.

Kadri, N., Agoub, M., El Gnaoui, S., Alami Kh, M., Hergueta, T., \& Moussaoui, D. (2005). Moroccan colloquial Arabic version of the MINI international neuropsychiatric interview (MINI): Qualitative and quantitative validation. European Psychiatry, 20(2), 193-195. https://doi.org/10.1016/j.eurpsy.2004.11.007.

Kay, S. R. (1991). Positive and negative syndromes in schizophrenia: Assessment and research. In Clinical and experimental research. New York: Monograph Series of the Department of Psychiatry Albert Einstein College of Medicine of Yeshiva University.

Kay, S. R., Fiszbein, A., \& Opler, L. A. (1987). The positive and negative syndrome scale (PANSS) for schizophrenia. Schizophrenia Bulletin, 13(2), 261-276.

Kay, S. R., Opler, L. A., Spitzer, R. L., Williams, J. B., Fiszbein, A., \& Gorelick, A. (1991). SCID-PANSS: Two-tier diagnostic system for psychotic disorders. Comprehensive Psychiatry, 32(4), 355-361.

Kazour, F., Zahreddine, N. R., Maragel, M. G., Almustafa, M. A., Soufia, M., Haddad, R., \& Richa, S. (2017). Post-traumatic stress disorder in a sample of Syrian refugees in Lebanon. Comprehensive Psychiatry, 72, 41-47. https://doi.org/10.1016/j.comppsych.2016.09.007.

Lancon, C., Reine, G., Llorca, P. M., \& Auquier, P. (1999). Validity and reliability of the French-language version of the positive and negative syndrome scale (PANSS). Acta Psychiatrica Scandinavica, 100(3), 237-243.

Leucht, S., Kane, J. M., Kissling, W., Hamann, J., Etschel, E., \& Engel, R. R. (2005). What does the PANSS mean? Schizophrenia Research, 79(2-3), 231-238. https://doi.org/10.1016/j.schres.2005.04.008.

Lindenmayer, J. P., Khan, A., Iskander, A., Abad, M. T., \& Parker, B. (2007). A randomized controlled trial of olanzapine versus haloperidol in the treatment of primary negative symptoms and neurocognitive deficits in schizophrenia. The Journal of Clinical Psychiatry, 68(3), 368-379.

Madhivanan, S., Jayaraman, K., Daniel, S. J., \& Ramasamy, J. (2017). Symptomatic remission in schizophrenia and its relationship with functional outcome measures in Indian population. Journal of Clinical and Diagnostic Research, 11(1), VC05-VC07. https://doi org/10.7860/JCDR/2017/22320.9242.

McGrath, J., Saha, S., Chant, D., \& Welham, J. (2008). Schizophrenia: A concise overview of incidence, prevalence, and mortality. Epidemiologic Reviews, 30, 67-76. https://doi.org/10.1093/epirev/ mxn001.

Mordal, J., Gundersen, O., \& Bramness, J. G. (2010). Norwegian version of the Mini-international neuropsychiatric interview: Feasibility, acceptability and test-retest reliability in an acute psychiatric ward. European Psychiatry, 25(3), 172-177. https://doi.org/10.1016/j. eurpsy.2009.02.004.

Ortiz, B. B., Araujo Filho, G. M., Araripe Neto, A. G., Medeiros, D., \& Bressan, R. A. (2013). Is disorganized schizophrenia a predictor of treatment resistance? Evidence from an observational study. Revista Brasileira de Psiquiatria, 35(4), 432-434. https://doi.org/10.1590/ 1516-4446-2013-1190.

Ortiz, B. B., Gadelha, A., Higuchia, C. H., Noto, C., Medeiros, D., Pitta, J. C., et al. (2015). Disorganized symptoms predicted worse functioning outcome in schizophrenia patients with established illness. Clinical Schizophrenia \& Related Psychoses, 1-18. https://doi.org/ 10.3371/CSRP.ORGA.022015.

Otsubo, T., Tanaka, K., Koda, R., Shinoda, J., Sano, N., Tanaka, S., et al. (2005). Reliability and validity of Japanese version of the Miniinternational neuropsychiatric interview. Psychiatry and Clinical Neurosciences, 59(5), 517-526. https://doi.org/10.1111/j.14401819.2005.01408.x.

Overall, J., \& Gorham, D. (1988). The brief psychiatric rating scale (BPRS): Recent developments in ascertainment and scaling. Psychopharmacology Bulletin, (24), 97-99.

Peralta Martin, V., \& Cuesta Zorita, M. J. (1994). [Validation of positive and negative symptom scale (PANSS) in a sample of Spanish schizophrenic patients]. Actas Luso-Españolas de Neurología, Psiquiatría y Ciencias Afines, 22(4), 171-177.

Phillips, M. R., Xiong, W., Wang, R. W., Gao, Y. H., Wang, X. Q., \& Zhang, N. P. (1991). Reliability and validity of the Chinese versions 
of the scales for assessment of positive and negative symptoms. Acta Psychiatrica Scandinavica, 84(4), 364-370.

Qatar Statistic Authority (2010). Census 2010. http://www.qsa.gov.qa/ QatarCensus/General_Results.aspx. Accessed March 2017.

Rossi, A., Alberio, R., Porta, A., Sandri, M., Tansella, M., \& Amaddeo, F. (2004). The reliability of the Mini-international neuropsychiatric interview-Italian version. Journal of Clinical Psychopharmacology, 24(5), 561-563.

Sheehan, D. V., Lecrubier, Y., Sheehan, K. H., Janavs, J., Weiller, E., Keskiner, A., et al. (1997). The validity of the MINI international neuropsychiatric interview (MINI) according to the SCID-P and its reliability. European Psychiatry, 12(5), 232-241. https://doi.org/10. 1016/S0924-9338(97)83297-X.

Sheehan, D. V., Lecrubier, Y., Sheehan, K. H., Amorim, P., Janavs, J., Weiller, E., et al. (1998). The Mini-international neuropsychiatric interview (M.I.N.I.): The development and validation of a structured diagnostic psychiatric interview for DSM-IV and ICD-10. J Clin Psychiatry, 59 Suppl 20, 22-33; quiz 34-57.

Spitzer, R. L., Williams, J. B., Gibbon, M., \& First, M. B. (1992). The structured clinical interview for DSM-III-R (SCID). I: History, rationale, and description. Archives of General Psychiatry, 49(8), 624629.

van Os, J., Burns, T., Cavallaro, R., Leucht, S., Peuskens, J., Helldin, L., et al. (2006). Standardized remission criteria in schizophrenia. Acta
Psychiatrica Scandinavica, 113(2), 91-95. https://doi.org/10.1111/j. 1600-0447.2005.00659.x.

Wittchen, H. U. (1994). Reliability and validity studies of the WHOcomposite international diagnostic interview (CIDI): A critical review. Journal of Psychiatric Research, 28(1), 57-84.

Yazbek, J. C., Haddad, R., Bou Khalil, R., Hlais, S., Rizk, G. A., Rohayem, J., \& Richa, S. (2014). Prevalence and correlates of alcohol abuse and dependence in Lebanon: Results from the Lebanese epidemiologic survey on alcohol (LESA). Journal of Addictive Diseases, 33(3), 221-233. https://doi.org/10.1080/10550887.2014. 950026.

Yehya, A., Ghuloum, S., Mahfoud, Z., Opler, M., Khan, A., Hammoudeh, S., et al. (2016). Validity and Reliability of the Arabic Version of the Positive and Negative Syndrome Scale. Psychopathology, 49(3), 181-187, doi:10.1159/000447328.

Yehya, A., Ghuloum, S., Mahfoud, Z., Opler, M., Khan, A., Hammoudeh, S., et al. (2017). Validation of the Five-Factor Model of the Arabic Version of the Positive and Negative Syndrome Scale in Schizophrenia. Psychopathology, doi:10.1159/000472154.

Publisher's Note Springer Nature remains neutral with regard to jurisdictional claims in published maps and institutional affiliations. 